\title{
LOCAL WEGNER AND LIFSHITZ TAILS ESTIMATES FOR THE DENSITY OF STATES FOR CONTINUOUS RANDOM SCHRÖDINGER OPERATORS
}

\author{
JEAN-MICHEL COMBES, FRANÇOIS GERMINET, AND ABEL KLEIN
}

\begin{abstract}
We introduce and prove local Wegner estimates for continuous generalized Anderson Hamiltonians, where the single-site random variables are independent but not necessarily identically distributed. In particular, we get Wegner estimates with a constant that goes to zero as we approach the bottom of the spectrum. As an application, we show that the (differentiated) density of states exhibits the same Lifshitz tails upper bound as the integrated density of states.
\end{abstract}

\section{INTRODUCTION}

In this paper we introduce and prove local Wegner estimates for continuous generalized Anderson Hamiltonians, where the single-site random variables are independent but not necessarily identically distributed. In particular, we get Wegner estimates with a constant that goes to zero as we approach the bottom of the spectrum. As an application of local Wegner estimates, we show that the (differentiated) density of states exhibits the same Lifshitz tails upper bound as the integrated density of states.

We consider continuous generalized Anderson Hamiltonians, which are random Schrödinger operators on $\mathrm{L}^{2}\left(\mathbb{R}^{d}\right)$ of the type

$$
H_{\boldsymbol{\omega}}:=-\Delta+V_{\mathrm{per}}+V_{\boldsymbol{\omega}},
$$

where: $\Delta$ is the $d$-dimensional Laplacian operator; $V_{\text {per }}$ is a bounded $q \mathbb{Z}^{d}$-periodic potential with $q \in \mathbb{N}$; and $V_{\boldsymbol{\omega}}$ is an alloy-type random potential:

$$
V_{\boldsymbol{\omega}}(x):=\sum_{j \in \mathbb{Z}^{d}} \omega_{j} u_{j}(x), \quad \text { with } \quad u_{j}(x)=u(x-j),
$$

where the single site potential $u$ is a nonnegative bounded measurable function on $\mathbb{R}^{d}$ with compact support, uniformly bounded away from zero in a neighborhood of the origin, and $\boldsymbol{\omega}=\left\{\omega_{j}\right\}_{j \in \mathbb{Z}^{d}}$ is a family of independent (not necessarily identical) random variables, such that, with $\mu_{j}$ denoting the probability distribution of $\omega_{j}$,

$$
\bigcup_{j \in \mathbb{Z}^{d}} \operatorname{supp} \mu_{j} \subset\left[M_{-}, M_{+}\right] \text {for some } \infty<M_{-}<M_{+}<\infty .
$$

Without loss of generality we specify $\|u\|_{\infty}=1$, which can be aways be achieved by rescaling the $\mu_{j}$. In this paper we assume that $\mu_{j}$ has no atoms (i.e., $\mu_{j}$ is a continuous measure) for all $j \in \mathbb{Z}^{d}$. The (ergodic) Anderson Hamiltonian is the special case when the $\left\{\omega_{j}\right\}_{j \in \mathbb{Z}^{d}}$ are identically distributed, i.e., $\mu_{j}=\mu$ for all $j \in \mathbb{Z}^{d}$.

2010 Mathematics Subject Classification. Primary 82B44; Secondary 47B80, 60H25, 81Q10.

A.K. was supported in part by the NSF under grants DMS-0457474 and DMS-1001509. 
Given a finite Borel measure $\nu$ on $\mathbb{R}$ and $s \geq 0$, we let $S_{\nu}(s):=\sup _{a \in \mathbb{R}} \nu([a, a+s])$, the concentration function of $\nu$, and set

$$
Q_{\nu}(s):=\left\{\begin{array}{ll}
\|\rho\|_{\infty} s & \text { if } \nu \text { has a bounded density } \rho \\
8 S_{\nu}(s) & \text { otherwise }
\end{array} .\right.
$$

$Q_{\nu}(s)$ is continuous on $[0, \infty$ [ if and only if the measure $\nu$ has no atoms, in which case $\lim _{s \downarrow 0} Q_{\nu}(s)=Q_{\nu}(0)=0[\mathrm{HT}$.

The finite volume operator $H_{\boldsymbol{\omega}}^{(\Lambda)}$, the restriction of $H_{\boldsymbol{\omega}}$ to a finite box $\Lambda$ with periodic boundary condition, has a finite number of eigenvalues in a given bounded interval $I \in \mathbb{R}$. Fluctuations of these eigenvalues due to the random variables $\left\{\omega_{j}\right\}_{j \in \mathbb{Z}^{d}}$ play a crucial role in the understanding of the localization properties of $H_{\boldsymbol{\omega}}$. When averaging over a single random variable, the fluctuations of the eigenvalues are controlled thanks to a spectral averaging principle: given a trace class operator $S \geq 0$, we have $\mathrm{CoH}$, $\mathrm{CoHK} 2$

$$
\mathbb{E}_{\omega_{j}}\left\{\operatorname{tr}\left\{\sqrt{u_{j}} \chi_{I}\left(H_{\boldsymbol{\omega}}^{(\Lambda)}\right) \sqrt{u_{j}} S\right\}\right\} \leq(\operatorname{tr} S) Q_{\mu_{j}}(|I|) \quad \text { for } \quad j \in \mathbb{Z}^{d} \cap \Lambda .
$$

Averaging over all the random variables, the expectation of the number of eigenvalues falling in an interval $I$ is controlled thanks to the celebrated Wegner estimate W, $\mathrm{CoH}, \mathrm{CoHK} 1, \mathrm{CoHK}$, $\mathrm{Kl} 2$ :

$$
\mathbb{E}\left\{\operatorname{tr} \chi_{I}\left(H_{\boldsymbol{\omega}}^{(\Lambda)}\right)\right\} \leq K_{W} Q_{\Lambda}(|I|)|\Lambda|,
$$

where

$$
Q_{\Lambda}(s):=\max _{j \in \Lambda \cap \mathbb{Z}^{d}} Q_{\mu_{j}}(s),
$$

and the constant $K_{W}$ depends on the parameters $d, u, M_{ \pm}$, and $\sup I$.

An estimate of the form

$$
\max _{j \in \Lambda \cap \mathbb{Z}^{d}} \mathbb{E}\left\{\operatorname{tr} \chi_{I}\left(H_{\boldsymbol{\omega}}^{(\Lambda)}\right) u_{j}\right\} \leq K_{L W} Q_{\Lambda}(|I|)
$$

will be called a local Wegner estimate. If the generalized Anderson Hamiltonian $H_{\boldsymbol{\omega}}$ satisfies the covering condition $\sum_{j \in \mathbb{Z}^{d} \cap \Lambda} u_{j} \geq C \chi_{\Lambda}>0$ with $C>0$, the Wegner estimate (1.6) can be immediately derived from the local Wegner estimate (1.8). If the random variables $\left\{\omega_{j}\right\}_{j \in \mathbb{Z}^{d}}$ are identically distributed, under the above covering condition it is equivalent to investigate local and global Wegner estimates. Indeed, using the covariance property of the model, in this case there exist constants $C_{1}$ and $C_{2}$ so that for any $j \in \Lambda$ we have

$$
\frac{C_{1}}{|\Lambda|} \mathbb{E}\left\{\operatorname{tr} \chi_{I}\left(H_{\boldsymbol{\omega}}^{(\Lambda)}\right)\right\} \leq \mathbb{E}\left\{\operatorname{tr} \chi_{I}\left(H_{\boldsymbol{\omega}}^{(\Lambda)}\right) u_{j}\right\} \leq \frac{C_{2}}{|\Lambda|} \mathbb{E}\left\{\operatorname{tr} \chi_{I}\left(H_{\boldsymbol{\omega}}^{(\Lambda)}\right)\right\} .
$$

In Theorem 2.3 we prove local Wegner estimates for generalized Anderson Hamiltonians with a covering condition. Moreover, we provide bounds on the local Wegner constant $K_{L W}$ in (1.8) that vanish as the energy approaches the bottom of the spectrum. These results are new, both in the ergodic and non-ergodic cases, and yield bounds on the density of states. Theorem 2.3 extends CoGK1, Lemma 4.1] to more general single site probability distributions and to a less restrictive covering condition.

Using a local Wegner estimate, we prove in Corollary 2.4 that the differentiated density of states exhibits the same Lifshitz tails upper bound as the integrated 
density of states for Anderson Hamiltonians with a covering condition and a singlesite probability distribution with a bounded density. This result had been shown to hold for discrete Anderson models [CoGK2].

\section{REsults}

We write

$$
\Lambda_{L}(x):=x+\left[-\frac{L}{2}, \frac{L}{2}\left[^{d}\right.\right.
$$

for the (half open-half closed) box of side $L>0$ centered at $x \in \mathbb{R}^{d}$. By $\Lambda_{L}$ we denote a box $\Lambda_{L}(x)$ for some $x \in \mathbb{R}^{d}$. Given a box $\Lambda=\Lambda_{L}(x)$, we set $\widetilde{\Lambda}=\Lambda \cap \mathbb{Z}^{d}$. If $B$ is a set, we write $\chi_{B}$ for its characteristic function. We set $\chi_{x}^{(L)}:=\chi_{\Lambda_{L}(x)}$, with $\chi_{x}:=\chi_{x}^{(1)}$ The Lebesgue measure of a Borel set $B \subset \mathbb{R}$ will be denoted by $|B|$. By a constant we will always mean a finite constant. Constants such as $C_{a, b, \ldots}$ will be finite and depending only on the parameters or quantities $a, b, \ldots$; they will be independent of other parameters or quantities in the equation. Note that $C_{a, b, \ldots}$ may stand for different constants in different sides of the same inequality.

Before stating our results, we normalize a generalized Anderson Hamiltonian $H_{\boldsymbol{\omega}}$ as follows. We first require $\inf _{j \in \mathbb{Z}^{d}} \inf \operatorname{supp} \mu_{j}=0$, which can always be realized by changing the periodic potential $V_{\text {per }}$. We then adjust $V_{\text {per }}$ by adding a constant so $\inf \sigma\left(-\Delta+V_{\text {per }}\right)=0$, in which case $\left[0, E_{*}\right] \subset \sigma\left(-\Delta+V_{\text {per }}\right)$ for some $E_{*}>0$. The result is a normalized generalized Anderson Hamiltonian as in the following definition, equal to the original generalized Anderson Hamiltonian given in (1.1)(1.2) plus a nonrandom constant. We also assume that the single site probability distributions have no atoms.

Definition 2.1. A normalized generalized Anderson Hamiltonian is a generalized Anderson Hamiltonian $H_{\boldsymbol{\omega}}$ as in (1.1) -(1.2), such that:

(i) The free Hamiltonian $H_{0}:=-\Delta+V_{\text {per }}$ has 0 as the bottom of its spectrum:

$$
\inf \sigma\left(H_{0}\right)=0 .
$$

(ii) The single site potential $u$ is a measurable function on $\mathbb{R}^{d}$ with

$$
\begin{array}{cc}
\|u\|_{\infty}=1 \text { and } & \left.u_{-} \chi_{\Lambda_{\delta_{-}}(0)} \leq u \leq \chi_{\Lambda_{\delta_{+}}(0)}, \text { where } u_{-}, \delta_{ \pm} \in\right] 0, \infty[ \\
\text { we set } & U_{+}:=\left\|\sum_{j \in \mathbb{Z}^{d}} u_{j}\right\|_{\infty} \leq \max \left\{1, \delta_{+}^{d}\right\} .
\end{array}
$$

(iii) $\boldsymbol{\omega}=\left\{\omega_{j}\right\}_{j \in \mathbb{Z}^{d}}$ is a family of independent random variables, such that for all $j \in \mathbb{Z}^{d}$ the probability distribution $\mu_{j}$ of $\omega_{j}$ has no atoms and

$$
0=\inf _{j \in \mathbb{Z}^{d}} \inf \operatorname{supp} \mu_{j}<M:=\sup _{j \in \mathbb{Z}^{d}} \sup \operatorname{supp} \mu_{j}<\infty .
$$

$H_{\boldsymbol{\omega}}$ is a normalized Anderson Hamiltonian if the $\left\{\omega_{j}\right\}_{j \in \mathbb{Z}^{d}}$ are identically distributed, i.e., $\mu_{j}=\mu$ for all $j \in \mathbb{Z}^{d}$. In this case $\mu$ is a probability measure with no atoms such that

$$
\{0, M\} \subset \operatorname{supp} \mu \subset[0, M], \text { where } M \in] 0, \infty[\text {. }
$$

Without loss of generality, we will always assume that a generalized Anderson Hamiltonian $H_{\boldsymbol{\omega}}$ is a normalized generalized Anderson Hamiltonian. In particular, Anderson Hamiltonians will also be understood to be normalized. 
We will need generalized Anderson Hamiltonians with more structure. We set

$$
\Gamma\left(j_{0}, K\right):=j_{0}+K \mathbb{Z}^{d}, \quad \text { where } j_{0} \in \mathbb{Z}^{d} \text { and } K \in \mathbb{N} \text {. }
$$

Note that for any $j \in \mathbb{Z}^{d}$ there exists $j^{\prime} \in \Gamma\left(j_{0}, K\right)$ such that $j \notin \Gamma\left(j^{\prime}, 2 K\right) \subset$ $\Gamma\left(j_{0}, K\right)$.

Definition 2.2. A generalized Anderson Hamiltonian $H_{\boldsymbol{\omega}}$ has a spine if there exist $j_{0} \in \mathbb{Z}^{d}$ and $K \in \mathbb{N}$ such that the random variables $\left\{\omega_{j}\right\}_{j \in \Gamma\left(j_{0}, K\right)}$ are identically distributed. In this case we will call $\Gamma=\Gamma\left(j_{0}, K\right)$ a spine of order $K$ for $H_{\boldsymbol{\omega}}$ and set $\mu_{\Gamma}:=\mu_{j}$ for $j \in \Gamma$.

An Anderson Hamiltonian $H_{\boldsymbol{\omega}}$ (in this language a generalized Anderson Hamiltonian with a spine of order 1 ) is a $q \mathbb{Z}^{d}$-ergodic family of random self-adjoint operators. It follows from standard results (cf. [KM1) that there exists fixed subsets $\Sigma$, $\Sigma_{\mathrm{pp}}, \Sigma_{\mathrm{ac}}$ and $\Sigma_{\mathrm{sc}}$ of $\mathbb{R}$ so that the spectrum $\sigma\left(H_{\boldsymbol{\omega}}\right)$ of $H_{\boldsymbol{\omega}}$, as well as its pure point, absolutely continuous, and singular continuous components, are equal to these fixed sets with probability one. With our normalization, the non-random spectrum $\Sigma$ of an Anderson Hamitonian $H_{\boldsymbol{\omega}}$ satisfies (cf. [KM2])

$$
\sigma\left(H_{0}\right) \subset \Sigma \subset[0, \infty[,
$$

with $\inf \Sigma=0$ and $\left[0, E_{*}\right] \subset \Sigma$ for some $E_{*}=E_{*}\left(V_{\text {per }}\right)>0$. Note that $\Sigma=$ $\sigma(-\Delta)=\left[0, \infty\left[\right.\right.$ if $V_{\text {per }}=0$.

A generalized Anderson Hamiltonian $H_{\boldsymbol{\omega}}$ is not, in general, an ergodic family of random self-adjoint operators, so the above considerations do not apply, and its spectrum is a random set. But it follows from Definition 2.1 that

$$
\sigma\left(H_{\boldsymbol{\omega}}\right) \subset[0, \infty[\text { with probability one. }
$$

Note furthermore that if the generalized Anderson Hamiltonian $H_{\boldsymbol{\omega}}$ has a spine $\Gamma$ of order $K$, then

$$
H_{\boldsymbol{\omega}_{\Gamma}}=H_{0}+V_{\boldsymbol{\omega}_{\Gamma}} \quad \text { where } \quad \boldsymbol{\omega}_{\Gamma}=\left\{\omega_{j}\right\}_{j \in \Gamma} \quad \text { and } \quad V_{\boldsymbol{\omega}_{\Gamma}}(x):=\sum_{j \in \Gamma} \omega_{j} u_{j}(x),
$$

is a $q K \mathbb{Z}^{d}$-ergodic family of random self-adjoint operators, and the above considerations for Anderson Hamiltonians apply. $\left(H_{\boldsymbol{\omega}_{\Gamma}}\right.$ is exactly like an Anderson Hamiltonian, except that the single site potentials are located in $K \mathbb{Z}^{d}$ instead of $\mathbb{Z}^{d}$.)

Let $H_{\boldsymbol{\omega}}$ be a generalized Anderson Hamiltonian. Finite volume operators are defined for finite boxes $\Lambda=\Lambda_{L}\left(j_{0}\right)$, where $j_{0} \in \mathbb{Z}^{d}$ and $L \in 2 q \mathbb{N}, L>\delta_{+}$. Given such $\Lambda$, we will consider the random Schrödinger operator $H_{\boldsymbol{\omega}}^{(\Lambda)}$ on $\mathrm{L}^{2}(\Lambda)$ given by the restriction of the generalized Anderson Hamiltonian $H_{\boldsymbol{\omega}}$ to $\Lambda$ with periodic boundary condition. To do so, we identify $\Lambda$ with a torus in the usual way by identifying opposite edges, and define finite volume operators

$$
H_{\omega}^{(\Lambda)}:=H_{0}^{(\Lambda)}+V_{\boldsymbol{\omega}}^{(\Lambda)} \quad \text { on } \quad \mathrm{L}^{2}(\Lambda) .
$$

The finite volume free Hamiltonian $H_{0}^{(\Lambda)}$ is given by

$$
H_{0}^{(\Lambda)}:=-\Delta^{(\Lambda)}+V_{\text {per }}^{(\Lambda)} \quad \text { on } \quad L^{2}(\Lambda),
$$

where $\Delta^{(\Lambda)}$ is the Laplacian on $\Lambda$ with periodic boundary condition and $V_{\text {per }}^{(\Lambda)}$ is the restriction of $V_{\text {per }}$ to $\Lambda$. The random potential $V_{\boldsymbol{\omega}}^{(\Lambda)}$ is the restriction of $V_{\boldsymbol{\omega}}^{(\Lambda)}$ 
to $\Lambda$, where, given $\boldsymbol{\omega}=\left\{\omega_{i}\right\}_{i \in \mathbb{Z}^{d}}$, we define $\boldsymbol{\omega}^{(\Lambda)}=\left\{\omega_{i}^{(\Lambda)}\right\}_{i \in \mathbb{Z}^{d}}$ by

$$
\omega_{i}^{(\Lambda)}=\omega_{i} \quad \text { if } \quad i \in \widetilde{\Lambda}, \quad \omega_{i}^{(\Lambda)}=\omega_{k}^{(\Lambda)} \quad \text { if } \quad k-i \in L \mathbb{Z}^{d} .
$$

Note that the random finite volume operator $H_{\omega}^{(\Lambda)}$ is not covariant with respect to translations in the torus unless $H_{\boldsymbol{\omega}}$ is an Anderson Hamiltonian.

Given $j \in \widetilde{\Lambda}$, we set

$$
u_{j}^{(\Lambda)}(x):=\sum_{k \in j+L \mathbb{Z}^{d}} u_{k}(x) \text { and } \chi_{j}^{(\Lambda)}(x):=\sum_{k \in j+L \mathbb{Z}^{d}} \chi_{k}(x) \text { for } x \in \Lambda,
$$

and rewrite $V_{\omega}^{(\Lambda)}$ as

$$
V_{\omega}^{(\Lambda)}=\sum_{j \in \widetilde{\Lambda}} \omega_{j} u_{j}^{(\Lambda)}
$$

We will often abuse the notation and just write $u_{j}$ and $\chi_{j}$ instead of $u_{j}^{(\Lambda)}$ and $\chi_{j}^{(\Lambda)}$ when working with finite volume operators. Note that

$$
\sum_{j \in \widetilde{\Lambda}} \chi_{j}^{(\Lambda)}(x)=1 \text { for all } x \in \Lambda .
$$

When the covering condition $\delta_{-} \geq 1$ (see (2.3) ) holds, we have

$$
\sum_{j \in \widetilde{\Lambda}} u_{j}^{(\Lambda)}(x) \geq u_{-} \text {for all } x \in \Lambda .
$$

Given a finite Borel measure $\nu$ on $\mathbb{R}$ with no atoms and finite moments, and $m \geq 1$, we set (recall (1.7))

$$
Q_{\nu}^{(m)}(s):=Q_{\nu^{(m)}}(s), \quad \text { where } \mathrm{d} \nu^{(m)}(t)=\left(1+|t|^{m}\right) \mathrm{d} \nu(t) .
$$

In particular, if $\operatorname{supp} \nu \subset[0, M]$ (cf. (2.5) ) we have

$$
Q_{\nu}^{(m)}(s) \leq(1+M)^{m} Q_{\nu}(s) \text { for } m \geq 1 .
$$

The finite Borel measure $\nu$ is said to be Hölder continuous of order $\alpha \in] 0,1]$ if there exists a constant $C_{\nu, \alpha}$ such that

$$
Q_{\nu}(s) \leq C_{\nu, \alpha} s^{\alpha} \text { for all } s \in[0,1] .
$$

If in addition $\operatorname{supp} \nu \subset[0, M]$, it follows that $\nu^{(m)}$ is also Hölder continuous of order $\alpha$ for all $m \geq 1$ :

$$
Q_{\nu}^{(m)}(s) \leq C_{\nu, \alpha, m} s^{\alpha} \quad \text { with } \quad C_{\nu, \alpha, m} \leq C_{\nu, \alpha}(1+M)^{m} .
$$

If $\nu$ has a bounded density $\rho$ (i.e., $\alpha=1$ ) and $\operatorname{supp} \nu \subset[0, M]$, then (2.20) holds with $C_{\nu, 1}=\|\rho\|_{\infty}$. In this case, for all $m \geq 1$ the measure $\nu^{(m)}$ has a bounded density $\rho^{(m)}(t)=\left(1+t^{m}\right) \rho(t)$, and

$$
Q_{\nu}^{(m)}(s) \leq\left\|\rho^{(m)}\right\|_{\infty} s \quad \text { with } \quad\left\|\rho^{(m)}\right\|_{\infty} \leq(1+M)^{m}\|\rho\|_{\infty} .
$$

Let $H_{\boldsymbol{\omega}}$ be a generalized Anderson Hamiltonian. If $B \subset \mathbb{R}$ is a Borel set, we write $P_{\boldsymbol{\omega}}^{(\Lambda)}(B):=\chi_{B}\left(H_{\boldsymbol{\omega}}^{(\Lambda)}\right)$ and $P_{\boldsymbol{\omega}}(B):=\chi_{B}\left(H_{\boldsymbol{\omega}}\right)$ for the spectral projections. Let $E_{0}>0, I \subset\left[0, E_{0}\right]$ an interval, and consider a box $\Lambda=\Lambda_{L}\left(j_{0}\right)$, where $L \in 2 q \mathbb{N}$, $L>\delta_{+}$, and $j_{0} \in \mathbb{Z}^{d}$. If $H_{\boldsymbol{\omega}}$ satisfies the covering condition $\delta_{-} \geq 1$ (see (2.3)), we have the Wegner estimate [CoH, CoHK2, CoGK1] (see also (2.27) below)

$$
\mathbb{E}\left\{\operatorname{tr} P_{\boldsymbol{\omega}}^{(\Lambda)}(I)\right\} \leq K_{W}\left(E_{0}\right) Q_{\Lambda}(|I|)|\Lambda| .
$$


Without assuming the covering condition, a careful reading of CoHK2, as in GKM, Appendix B], gives

$$
\mathbb{E}\left\{\operatorname{tr} P_{\boldsymbol{\omega}}^{(\Lambda)}(I)\right\} \leq K_{W}\left(E_{0}\right) Q_{\Lambda}^{\left(m_{d}\right)}(|I|)|\Lambda| \quad \text { with } \quad m_{d}=2^{2+\frac{\log d}{\log 2}},
$$

where

$$
Q_{\Lambda}^{(m)}(s):=\max _{j \in \widetilde{\Lambda}} Q_{\mu_{j}}^{(m)}(s) \leq(1+M)^{m} Q_{\Lambda}(|I|) \quad \text { for } \quad m \geq 1 .
$$

The constants $K_{W}\left(E_{0}\right)$ in (2.23) and (2.24) depend only on $d, V_{\text {per }}, \delta_{+}, u_{-}$; they do not depend on the probability distributions $\mu_{j}$. If the generalized Anderson Hamiltonian $H_{\boldsymbol{\omega}}$ has a spine $\Gamma$ we set $Q_{\Gamma}^{(m)}=Q_{\mu_{\Gamma}}^{(m)}$.

We now state our local Wegner estimates. We set $\left[\left[\frac{d}{4}\right]\right]=\left\lfloor\frac{d}{4}\right\rfloor+1$, the smallest integer $>\frac{d}{4}$.

Theorem 2.3. Let $H_{\boldsymbol{\omega}}$ be a generalized Anderson Hamiltonian with $\delta_{-} \geq 1$, and let $\Lambda=\Lambda_{L}\left(j_{0}\right)$, where $L \in 2 q \mathbb{N}, L>\delta_{+}$, and $j_{0} \in \mathbb{Z}^{d}$.

(i) Given $E_{0}>0$, for all intervals $I \subset\left[0, E_{0}\right]$ we have

$$
\max _{j \in \tilde{\Lambda}} \mathbb{E}\left\{\operatorname{tr} P_{\boldsymbol{\omega}}^{(\Lambda)}(I) u_{j}^{(\Lambda)}\right\} \leq C_{d,\left\|V_{\text {per }}^{-}\right\|, \delta_{+}} u_{-}^{-\frac{3}{2}}\left(1+E_{0}\right)^{2\left[\left[\frac{d}{4}\right]\right]}\left(1+\log \left(1+E_{0}\right)\right) Q_{\Lambda}(|I|),
$$

yielding the Wegner estimate

$$
\mathbb{E}\left\{\operatorname{tr} P_{\omega}^{(\Lambda)}(I)\right\} \leq C_{d,\left\|V_{\text {per }}^{-}\right\|, \delta_{+}} u_{-}^{-\frac{5}{2}}\left(1+E_{0}\right)^{2\left[\left[\frac{d}{4}\right]\right]}\left(1+\log \left(1+E_{0}\right)\right) Q_{\Lambda}(|I|)|\Lambda| .
$$

(ii) Suppose the generalized Anderson Hamiltonian $H_{\boldsymbol{\omega}}$ has a spine $\Gamma$ of order $K$. Given $\eta \in] 0, \frac{d}{2}\left[\right.$, there exists $E_{1}=E_{1}\left(\eta, d, V_{\text {per }}, \delta_{ \pm}, u_{-}, \mu_{\Gamma}, K\right)>0$, such that for all $\left.E_{0} \in\right] 0, E_{1}\left[\right.$ and intervals $I \subset\left[0, E_{0}\right]$, we have

$$
\max _{j \in \tilde{\Lambda}} \mathbb{E}\left\{\operatorname{tr} P_{\boldsymbol{\omega}}^{(\Lambda)}(I) u_{j}^{(\Lambda)}\right\} \leq \mathrm{e}^{-E_{0}^{-\frac{d}{2}+\eta}} Q_{\Lambda}(|I|),
$$

yielding the Wegner estimate

$$
\mathbb{E}\left\{\operatorname{tr} P_{\omega}^{(\Lambda)}(I)\right\} \leq u_{-}^{-1} \mathrm{e}^{-E_{0}^{-\frac{d}{2}+\eta}} Q_{\Lambda}(|I|)|\Lambda|,
$$

for $L$ large (how large depending on $E_{0}, d, V_{\mathrm{per}}, \delta_{ \pm}, u_{-}, \mu_{\Gamma}, K, \eta$ ).

(iii) Suppose the generalized Anderson Hamiltonian $H_{\omega}$ has a spine $\Gamma$ of order $K$ with a Hölder continuous single-site probability distribution $\mu_{\Gamma}$ of order $\alpha$, and let $L \in 2 q K \mathbb{N}$. Then there exists $E_{1}=E_{1}\left(d, V_{\mathrm{per}}, \delta_{ \pm}, u_{-}, \mu_{\Gamma}, K\right)>$ 0 , such that for all $\left.E_{0} \in\right] 0, E_{1}\left[\right.$, intervals $I \subset\left[0, E_{0}\right]$, and $\left.\eta \in\right] 0,1[$, we have

$$
\begin{aligned}
\max _{j \in \widetilde{\Lambda}} \mathbb{E}\left\{\operatorname{tr} P_{\omega}^{(\Lambda)}(I) u_{j}^{(\Lambda)}\right\} & \leq C_{\eta}\left(C_{\mu_{\Gamma}}\left(2 \alpha E_{0} \log \frac{1}{2 \alpha E_{0} C_{\mu_{\Gamma}}^{\frac{1}{\alpha}}}\right)^{\alpha}\right)^{1-\eta} Q_{\Lambda}(|I|) \\
& \leq C_{\eta, \mu_{\Gamma}} E_{0}^{\alpha\left(1-\frac{\eta}{2}\right)} Q_{\Lambda}(|I|),
\end{aligned}
$$

yielding the Wegner estimate

$$
\mathbb{E}\left\{\operatorname{tr} P_{\boldsymbol{\omega}}^{(\Lambda)}(I)\right\} \leq C_{\eta, \mu_{\Gamma}} u_{-}^{-1} E_{0}^{\alpha\left(1-\frac{\eta}{2}\right)} Q_{\Lambda}(|I|)|\Lambda|,
$$

for $L$ large (how large depending on $d, V_{\mathrm{per}}, \delta_{ \pm}, u_{-}, \mu_{\Gamma}, K$ ), where $C_{\eta}=$ $C_{d, V_{\mathrm{per}}, \delta_{ \pm}, u_{-}, K, E_{1}, \eta}, C_{\mu_{\Gamma}}=C_{\mu_{\Gamma}, \alpha, m_{d}}$ as in (2.21) with $m_{d}$ given in (2.24), and $C_{\eta, \mu_{\Gamma}}=C_{d, V_{\mathrm{per}}, \delta_{ \pm}, u_{-}, K, E_{1}, \mu_{\Gamma}, \eta}$. 
Part (i), namely (2.26), gives a local version of of the Wegner estimates (2.23) and (2.25). It is of the form given in (1.8), valid at all energies $E_{0}$ with a constant $K_{L W}=K_{L W}\left(E_{0}\right)$, but the constant does not get small as $E_{0} \downarrow 0$. Parts (ii) and (iii) provide local Wegner estimates valid for small $E_{0}$ with $\lim _{E_{0} \downarrow 0} K_{L W}\left(E_{0}\right)=0$.. Part (ii) requires less hypotheses, and seems to provide a stronger result. But we believe that the energy interval $\left[0, E_{1}\right]$ where the estimates hold is bigger in (iii). The proof of (ii) takes advantage of the Lifshitz tails estimate, and is thus valid in an energy interval at the bottom of the spectrum where we have Lifshitz tails. The proof of (iii) uses dynamical localization estimates, and is valid in the energy interval where we can perform the bootstrap multiscale analysis of GK1, which in principle is larger than the region of Lifshitz tails. In addition, (2.30), unlike (2.28), shows the explicit dependence of the constant on the single-site probability distribution $\mu_{\Gamma}$. (This is the reason why we state (2.30) in addition to (2.31).) Note that when $\mu_{\Gamma}$ has a bounded density $\rho$, we have (recall (2.22) $) C_{\mu_{\Gamma}}=\left\|\rho^{\left(m_{d}\right)}\right\|_{\infty} \leq(1+M)^{m_{d}}\|\rho\|_{\infty}$.

The Wegner estimates (2.29) and (2.32), with constants that go to zero as $E_{0} \rightarrow$ 0 , only require the covering condition $\delta_{-} \geq 1$. This is a substantial improvement on the similar Wegner estimate given in [CoGK1, Lemma 4.1(i)], which requires the double covering condition $\delta_{-} \geq 2$.

An Anderson Hamiltonian $H_{\boldsymbol{\omega}}$ satisfies a Lifshitz tails estimate, which asserts that its integrated density of states $N(E)$ has exponential fall off as the energy $E$ approaches the bottom of the spectrum. The finite volume operator $H_{\boldsymbol{\omega}}^{(\Lambda)}$ has a compact resolvent, and hence its ( $\boldsymbol{\omega}$-dependent) spectrum consists of isolated eigenvalues with finite multiplicity. We recall that the integrated density of states (IDS) for $H_{\boldsymbol{\omega}}$ is given, for a.e. $E \in \mathbb{R}$, by $\left(\Lambda_{L}=\Lambda_{L}(0)\right)$

$$
N(E):=\lim _{L \rightarrow \infty}\left|\Lambda_{L}\right|^{-1} \operatorname{tr} \chi_{]-\infty, E]}\left(H_{\boldsymbol{\omega}}^{\left(\Lambda_{L}\right)}\right) \quad \text { for } \mathbb{P} \text {-a.e. } \boldsymbol{\omega}
$$

in the sense that the limit exists and is the same for $\mathbb{P}$-a.e. $\boldsymbol{\omega}$ (cf. $\mathrm{CL}, \mathbb{N}, \mathrm{PF}$ ). Recalling that with our normalization the bottom of the spectrum is at 0 , the IDS satisfies the Lifshitz tails estimate (e.g., Klo1, Corollary 2.2 and Remark 7.1])

$$
\lim _{E \downarrow 0} \frac{\log |\log N(E)|}{\log E} \leq-\frac{d}{2}
$$

Equality is actually known to hold in (2.34).

Since the integrated density of states $N(E)$ is an increasing function, it has a derivative $n(E):=N^{\prime}(E) \geq 0$ almost everywhere, the density of states. Note that by ergodicity with respect to $q \mathbb{Z}^{d}$ we have

$$
N(E)=q^{-d} \mathbb{E}\left\{\operatorname{tr} \chi_{0}^{(q)} \chi_{]-\infty, E]}\left(H_{\boldsymbol{\omega}}\right) \chi_{0}^{(q)}\right\},
$$

and hence

$$
N\left(E^{\prime}\right)-N(E) \leq q^{-d} \mathbb{E}\left\{\operatorname{tr} \chi_{0}^{(q)} \chi_{] E, E^{\prime}\right]}\left(H_{\boldsymbol{\omega}}\right) \chi_{0}^{(q)}\right\} \quad \text { for } \quad E \leq E^{\prime} .
$$

As a consequence, if the single-site probability distribution $\mu$ has a bounded density $\rho$, and the local Wegner estimate (1.8) holds for intervals $I \subset\left[0, E_{0}\right]$, we conclude that

$$
n(E) \leq q^{-d} K_{L W}\|\rho\|_{\infty} \quad \text { for a.e. } \quad E \in\left[0, E_{0}\right] .
$$

The following corollary, which provides an exponentially small bound for the density of states within the regime of Lifshitz tails, is an immediate corollary of Theorem 2.3(ii), using (2.28) and (2.37). 
Corollary 2.4. Let $H_{\boldsymbol{\omega}}$ be an Anderson Hamiltonian with $\delta_{-} \geq 1$, whose singlesite probability distribution $\mu$ has a bounded density $\rho$. Then there exists a Borel set $\mathcal{N} \subset[0,1]$ of zero Lebesgue measure such that

$$
\lim _{E \downarrow 0 ; E \notin \mathcal{N}} \frac{\log |\log n(E)|}{\log E} \leq-\frac{d}{2} .
$$

The same Lifshitz tails estimate for the density of states holds for the discrete Anderson model [CoGK2].

\section{Proof of local Wegner estimates}

\subsection{A simple Lemma.}

Lemma 3.1. Let $H=H_{0}+W$, where $H, H_{0}$ are semi-bounded self-adjoint operators, say $H, H_{0} \geq-\Theta$ for some $\Theta>0$, such that $(H+\Theta+1)^{-p}$ is a trace class operator for some $p>0$, and $W$ is a bounded self-adjoint operator. Let $E_{0} \in \mathbb{R}$. Let $f, h$ be bounded Borel measurable nonnegative functions with compact support such that $f=\chi_{\left(-\infty, E_{0}\right]} f, h=\chi_{\left[E_{0}, \infty\right)} h$, and $H_{0} h\left(H_{0}\right)$ is a bounded operator. Then $f(H) W h\left(H_{0}\right)$ is trace class and

$$
\operatorname{tr} f(H) W h\left(H_{0}\right) \leq 0 .
$$

In particular, if $f, g$ are bounded Borel measurable nonnegative functions such that $f=\chi_{\left(-\infty, E_{0}\right]} f$ and $\chi_{\left(-\infty, E_{0}\right]} \leq g \leq 1$, we have $f(H) W$ and $f(H) W g\left(H_{0}\right)$ trace class, and

$$
\operatorname{tr} f(H) W \leq \operatorname{tr} f(H) W g\left(H_{0}\right) .
$$

Note that $W$ does not need to be positive.

Proof. Let $f, h$ be as above, note that $f(H)$ is trace class. Then, as $W=H-H_{0}$, we have

$$
\operatorname{tr} f(H) W h\left(H_{0}\right)=\operatorname{tr} f(H) H h\left(H_{0}\right)-\operatorname{tr} f(H) H_{0} h\left(H_{0}\right),
$$

where both $f(H) H h\left(H_{0}\right)$ and $f(H) H_{0} h\left(H_{0}\right)$ are trace class operators. Moreover,

$$
\begin{aligned}
\operatorname{tr} f(H) H h\left(H_{0}\right) & \leq E_{0} \operatorname{tr} f(H) h\left(H_{0}\right), \\
\operatorname{tr} f(H) H_{0} h\left(H_{0}\right) & \geq E_{0} \operatorname{tr} f(H) h\left(H_{0}\right),
\end{aligned}
$$

so (3.1) follows.

Now let $f, g$ be as above. Let also $\chi_{n}=\chi_{(-\infty, n]}$. Then, using (3.1),

$$
\begin{aligned}
\operatorname{tr} f(H) W & =\lim _{n \rightarrow \infty} \operatorname{tr} f(H) W \chi_{n}\left(H_{0}\right) \\
& =\operatorname{tr} f(H) W g\left(H_{0}\right)+\lim _{n \rightarrow \infty} \operatorname{tr} f(H) W \chi_{n}\left(H_{0}\right)\left(1-g\left(H_{0}\right)\right) \\
& \leq \operatorname{tr} f(H) W g\left(H_{0}\right) .
\end{aligned}
$$

3.2. Norms on random operators. Given $p \in[1, \infty), \mathcal{T}_{p}$ will denote the Banach space of bounded operators $S$ on $\mathrm{L}^{2}\left(\mathbb{R}^{d}, \mathrm{~d} x\right)$ with $\|S\|_{\mathcal{T}_{p}}=\|S\|_{p}:=\left(\operatorname{tr}|S|^{p}\right)^{\frac{1}{p}}<\infty$. A random operator $S_{\omega}$ is a strongly measurable map from the probability space $(\Omega, \mathbb{P})$ to bounded operators on $\mathrm{L}^{2}\left(\mathbb{R}^{d}, \mathrm{~d} x\right)$. Given $p \in[1, \infty)$, we set

$$
\left|\left\|\left|S_{\omega}\right|\right\|_{p}:=\left\{\mathbb{E}\left\{\left\|S_{\omega}\right\|_{p}^{p}\right\}\right\}^{\frac{1}{p}}=\|\| S_{\omega}\left\|_{\mathcal{T}_{p}}\right\|_{L^{p}(\Omega, \mathbb{P})},\right.
$$

and

$$
\left|\left\|\left|S_{\omega}\right|\right\|\right|_{\infty}:=\|\| S_{\omega}\|\|_{L^{\infty}(\Omega, \mathbb{P})} .
$$


These are norms on random operators, note that

$$
\left|\left\|\left|S_{\omega}\right|\right\|\right|_{q} \leq\left|\left\|\left|S_{\omega}\right|\right\|\right|_{\infty}^{\frac{q-p}{q}}\left|\left\|\left|S_{\omega}\right|\right\|\right|_{p}^{\frac{p}{q}} \quad \text { for } 1 \leq p \leq q<\infty,
$$

and they satisfy Holder's inequality:

$$
\left|\left\|\left|S_{\omega} T_{\omega}\right|\right\|\right|_{r} \leq\left|\left\|\left|S_{\omega}\right|\right\|\right|_{p}\left|\left\|T_{\omega}|\||_{q} \quad \text { for } r, p, q \in[1, \infty] \text { with } \frac{1}{r}=\frac{1}{p}+\frac{1}{q} .\right.\right.
$$

\subsection{Proof of Theorem 2.3 ,}

Proof. Let $H_{\boldsymbol{\omega}}$ be a generalized Anderson Hamiltonian satisfying the covering condition $\delta_{-} \geq 1$ (see (2.3)), and consider a box $\Lambda=\Lambda_{L}$, where $L \in 2 q \mathbb{N}, L>\delta_{+}$. Let $E_{0}>0, I \subset\left[0, E_{0}\right]$ an interval. Let $g$ be a bounded Borel measurable function such that $\chi_{\left(-\infty, E_{0}\right]} \leq g \leq 1$. Given $j \in \mathbb{Z}^{d}$, we let $\boldsymbol{\omega}_{j}^{\perp}=\left\{\omega_{k}\right\}_{k \in \mathbb{Z}^{d} \backslash\{j\}}$, write $\boldsymbol{\omega}=\left(\boldsymbol{\omega}_{j}^{\perp}, \omega_{j}\right)$, and consider the random Schrödinger operator $H_{\boldsymbol{\omega}_{j}^{\perp}}=H_{\boldsymbol{\omega}}-\omega_{j} u_{j}$.

To simplify the notation, we will write $u_{k}$ and $\chi_{k}$ for $u_{k}^{(\Lambda)}$ and $\chi_{k}^{(\Lambda)}$, and set

$$
\begin{aligned}
\hat{\chi}_{k} & :=u_{k}^{-\frac{1}{2}} \chi_{k} \leq u_{-}^{-\frac{1}{2}} \chi_{k} \quad \text { for } \quad k \in \widetilde{\Lambda} \quad\left(\text { recall (2.3) and } \delta_{-} \geq 1\right), \\
P & =P_{\boldsymbol{\omega}}^{(\Lambda)}(I):=\chi_{I}\left(H_{\boldsymbol{\omega}}^{(\Lambda)}\right), \\
\widetilde{P}_{j} & =\widetilde{P}_{j, \boldsymbol{\omega}_{j}^{\perp}}^{(\Lambda)}(I):=g\left(H_{\boldsymbol{\omega}_{j}^{\perp}}^{(\Lambda)}\right), \quad \text { where } \quad j \in \widetilde{\Lambda} \quad \text { and } \quad H_{\boldsymbol{\omega}_{j}^{\perp}}^{(\Lambda)}=H_{\boldsymbol{\omega}}^{(\Lambda)}-\omega_{j} u_{j} .
\end{aligned}
$$

Given $j \in \widetilde{\Lambda}$, it follows from Lemma 3.1, using (2.16), that

$$
\begin{aligned}
\operatorname{tr} P u_{j} & \leq \operatorname{tr} P u_{j} \widetilde{P}_{j}=\sum_{k \in \widetilde{\Lambda}} \operatorname{tr} P u_{j} \widetilde{P}_{j} \chi_{k}=\sum_{k \in \widetilde{\Lambda}} \operatorname{tr} u_{k}^{\frac{1}{2}} P u_{j}^{\frac{1}{2}} u_{j}^{\frac{1}{2}} \widetilde{P}_{j} \hat{\chi}_{k} \\
& =\sum_{k \in \widetilde{\Lambda}} \operatorname{tr} u_{k}^{\frac{1}{2}} P u_{j}^{\frac{1}{2}} T_{j, k},
\end{aligned}
$$

where

$$
T_{j, k}=u_{j}^{\frac{1}{2}} \widetilde{P}_{j} \hat{\chi}_{k}
$$

It follows that

$$
\begin{aligned}
& \mathbb{E}\left\{\operatorname{tr} P u_{j}\right\}=\|\| P u_{j}^{\frac{1}{2}}\left|\| | _ { 2 } ^ { 2 } \leq \sum _ { k \in \widetilde { \Lambda } } \| \left\|u_{k}^{\frac{1}{2}} P u_{j}^{\frac{1}{2}} T_{j, k}|\||_{1}\right.\right. \\
& \leq \sum_{k \in \widetilde{\Lambda}}|||| u_{k}^{\frac{1}{2}} P\left|\left\|| _ { 2 } \left|\left\|| | P u _ { j } ^ { \frac { 1 } { 2 } } T _ { j , k } \left|\left\|\left.\right|_{2} \leq\left(\operatorname { m a x } _ { r \in \widetilde { \Lambda } } | \| | | P u _ { r } ^ { \frac { 1 } { 2 } } | \| | _ { 2 } ) \sum _ { k \in \widetilde { \Lambda } } | \left\||| P u_{j}^{\frac{1}{2}} T_{j, k}|\||_{2},\right.\right.\right.\right.\right.\right.\right.\right.
\end{aligned}
$$

and hence

$$
\max _{r \in \widetilde{\Lambda}}\left|\left\|\left|P u_{r}^{\frac{1}{2}}\right|\right\|\right|_{2} \leq \max _{j \in \widetilde{\Lambda}} \sum_{k \in \widetilde{\Lambda}}\|\| P u_{j}^{\frac{1}{2}} T_{j, k} \mid\|\|_{2} .
$$

We have

$$
\begin{aligned}
\|\| P u_{j}^{\frac{1}{2}} T_{j, k} \mid \|_{2}^{2} & =\mathbb{E}\left\{\operatorname{tr}\left\{P u_{j}^{\frac{1}{2}} T_{j, k} T_{j, k}^{*} u_{j}^{\frac{1}{2}} P\right\}\right\}=\mathbb{E}\left\{\operatorname{tr}\left\{u_{j}^{\frac{1}{2}} P u_{j}^{\frac{1}{2}} T_{j, k} T_{j, k}^{*}\right\}\right\} \\
& \leq Q_{\mu_{j}}(|I|) \mathbb{E}_{\boldsymbol{\omega}_{j}^{\perp}}\left\{\operatorname{tr} T_{j, k} T_{j, k}^{*}\right\}=Q_{\mu_{j}}(|I|)\|\| T_{j, k} \mid \|_{2}^{2},
\end{aligned}
$$


where we used the basic spectral averaging estimate (1.5) (note that $T_{j, k}$ does not depend on $\omega_{j}$ ). It follows that

$$
\max _{r \in \widetilde{\Lambda}} \mathbb{E}\left\{\operatorname{tr} P u_{r}\right\} \leq Q_{\Lambda}(|I|)\left(\left.\max _{j \in \widetilde{\Lambda}} \sum_{k \in \widetilde{\Lambda}}|\|| T_{j, k}||\right|_{2}\right)^{2} .
$$

To prove (i), we use (3.9) with

$$
\left|\left\|\left|T_{j, k}\right|\right\|\right|_{1} \leq u_{-}^{-1}\left|\left\|\left|\widetilde{P}_{j} u_{j}^{\frac{1}{2}}\right|\right\|\right|_{2}\left|\left\||| \widetilde{P}_{j} u_{k}^{\frac{1}{2}} \mid\right\| \|_{2} \leq u_{-}^{-1} \max _{r \in \bar{\Lambda}} \mathbb{E}\left\{\operatorname{tr} \widetilde{P}_{j} u_{r}\right\},\right.
$$

to conclude that

$$
\max _{r \in \widetilde{\Lambda}} \mathbb{E}\left\{\operatorname{tr} P u_{r}\right\} \leq u_{-}^{-1} Q_{\Lambda}(|I|)\left(\max _{j, k \in \widetilde{\Lambda}} \mathbb{E}\left\{\operatorname{tr} \widetilde{P}_{j} u_{k}\right\}\right)\left(\max _{j \in \widetilde{\Lambda}} \sum_{k \in \widetilde{\Lambda}}\left|\left\|\left|T_{j, k}\right|\right\|\right|_{\infty}^{\frac{1}{2}}\right)^{2} .
$$

If the function $g$ in (3.13) satisfies $g(E)=0$ for $E>E_{1} \geq E_{0}$, it follows from the usual trace estimate for Schrödinger operators (e.g., GK3, Lemma A.4]) that

$$
\operatorname{tr} \widetilde{P}_{j} u_{k} \leq C_{d,\left\|V_{\text {per }}^{-}\right\|, \delta_{+}}\left(1+E_{1}\right)^{2\left[\left[\frac{d}{4}\right]\right]} \quad \text { for all } j, k \in \widetilde{\Lambda} \text { and } \boldsymbol{\omega} \in\left[0, \infty\left[^{\mathbb{Z}^{d}},\right.\right.
$$

where $V_{\text {per }}^{-}$denotes the negative part of $V_{\text {per }}$ and $\left[\left[\frac{d}{4}\right]\right]$ is the smallest integer $>\frac{d}{4}$. We now take $g(E)=g_{0}\left(E-E_{0}\right)$, where $g_{0} \in C^{\infty}(\mathbb{R}), 0 \leq g_{0} \leq 1, g_{0}(E)=1$ for $E \leq 0$, and $g_{0}(E)=0$ for $E \geq 1$. We now apply [GK2, Theorem 2], concluding that that for all $n \in \mathbb{N}, j, k \in \widetilde{\Lambda}$, and $\boldsymbol{\omega} \in\left[0, \infty\left[\mathbb{Z}^{d}\right.\right.$ we have

$$
\left\|T_{j, k}\right\| \leq u_{-}^{-\frac{1}{2}}\left\|u_{j}^{\frac{1}{2}} \widetilde{P}_{j} \chi_{k}\right\| \leq u_{-}^{-\frac{1}{2}}\left\|\chi_{\Lambda_{\delta_{+}}(j)} \widetilde{P}_{j} \chi_{k}\right\| \leq C_{d,\left\|V_{\mathrm{per}}^{-}\right\|, \delta_{+}, n} u_{-}^{-\frac{1}{2}} \frac{1+\log \left(1+E_{0}\right)}{\left(1+d_{\Lambda}(j, k)\right)^{n}},
$$

where $d_{\Lambda}($,$) is the distance on the torus \Lambda=\Lambda_{L}$ :

$$
d_{\Lambda}\left(y, y^{\prime}\right)=\min _{r \in L \mathbb{Z}^{d}}\left|y-y^{\prime}+r\right| \quad \text { for } \quad y, y^{\prime} \in \Lambda .
$$

(Note that the results in GK2] are valid on the torus with the appropriate modifications, the main one being the use of the distance on the torus.) Taking $n=2 d+2$, and using

$$
\sum_{k \in \widetilde{\Lambda}}\left(1+d_{\Lambda}(j, k)\right)^{-(d+1)} \leq \sum_{k \in \mathbb{Z}^{d}}(1+|k|)^{-(d+1)}<\infty \quad \text { for all } \quad j \in \widetilde{\Lambda},
$$

we conclude that

$$
\left(\max _{j \in \widetilde{\Lambda}} \sum_{k \in \widetilde{\Lambda}}\left|\left\|\left|T_{j, k}\right|\right\|\right|_{\infty}^{\frac{1}{2}}\right)^{2} \leq C_{d,\left\|V_{\text {per }}^{-}\right\|, \delta_{+}} u_{-}^{-\frac{1}{2}}\left(1+\log \left(1+E_{0}\right)\right) .
$$

It now follows from (3.21), (3.22), and (3.26) that

$$
\max _{r \in \widetilde{\Lambda}} \mathbb{E}\left\{\operatorname{tr} P u_{r}\right\} \leq C_{d,\left\|V_{\text {per }}^{-}\right\|, \delta_{+}}\left(1+E_{0}\right)^{2\left[\left[\frac{d}{4}\right]\right]}\left(1+\log \left(1+E_{0}\right)\right) u_{-}^{-\frac{3}{2}} Q_{\Lambda}(|I|),
$$

which is (2.26). The Wegner estimate (2.27) is an immediate consequence of (2.26) and (2.17). This finishes the proof of (i). 
Now suppose that the generalized Anderson Hamiltonian $H_{\boldsymbol{\omega}}$ has a spine $\Gamma$ of order $K$. For any $j \in \mathbb{Z}^{d}$ there exists a spine $\Gamma_{j} \subset \Gamma$ of order $2 K$ with $j \notin \Gamma_{j}$, and we can write

$$
H_{\boldsymbol{\omega}_{j}^{\perp}}=H_{\boldsymbol{\omega}_{\Gamma_{j}}}+V_{\boldsymbol{\omega}_{j}^{\perp} \backslash \boldsymbol{\omega}_{\Gamma_{j}}}, \quad \text { where } \quad 0 \leq V_{\boldsymbol{\omega}_{j}^{\perp} \backslash \boldsymbol{\omega}_{\Gamma_{j}}}:=V_{\boldsymbol{\omega}_{j}^{\perp}}-V_{\boldsymbol{\omega}_{\Gamma_{j}}} \leq U_{+} .
$$

We take the function $g$ in (3.13) so $0 \leq g \leq 1, g(E)=1$ for $E \leq E_{0}, g(E)=0$ for $E \geq E^{*}$; where $E^{*} \geq E_{0}$ will be later chosen appropriately. We have (writing $H_{\boldsymbol{\omega}_{j}^{\perp}}$ for $H_{\boldsymbol{\omega}_{j}^{\perp}}^{(\Lambda)}$, etc.)

$$
\operatorname{tr}\left\{\widetilde{P}_{j} u_{k}\right\} \leq \mathrm{e}^{t E^{*}} \operatorname{tr}\left\{\mathrm{e}^{-t H_{\omega_{j}}^{\perp}} u_{k}\right\} \leq \mathrm{e}^{t E^{*}} \operatorname{tr}\left\{\mathrm{e}^{-t H_{\omega_{\Gamma_{j}}}} u_{k}\right\} \quad \text { for } \quad t>0,
$$

where we used (3.28) and the positivity preserving property as in [BoGKS, Lemma 2.2]. Setting

$$
P_{\boldsymbol{\omega}_{\Gamma_{j}}}([0, E]):=\chi_{[0, E]}\left(H_{\boldsymbol{\omega}_{\Gamma_{j}}}^{(\Lambda)}\right)=\chi_{]-\infty, E]}\left(H_{\boldsymbol{\omega}_{\Gamma_{j}}}^{(\Lambda)}\right)
$$

we get, again using the positivity preserving property as in [BoGKS, Lemma 2.2], and requiring $t \geq 2$, that for all $E>0$ we have

$$
\begin{aligned}
\operatorname{tr}\left\{\mathrm{e}^{-t H_{\boldsymbol{\omega}_{\Gamma_{j}}}} u_{k}\right\} & \leq \operatorname{tr}\left\{P_{\boldsymbol{\omega}_{\Gamma_{j}}}([0, E]) u_{k}\right\}+\mathrm{e}^{-\frac{t}{2} E} \operatorname{tr}\left\{\mathrm{e}^{-\frac{t}{2} H_{\boldsymbol{\omega}_{\Gamma_{j}}}} u_{k}\right\} \\
& \leq \operatorname{tr}\left\{P_{\boldsymbol{\omega}_{\Gamma_{j}}}([0, E]) u_{k}\right\}+\mathrm{e}^{-\frac{t}{2} E} \operatorname{tr}\left\{\mathrm{e}^{-\frac{t}{2} H_{0}} u_{k}\right\} \\
& \leq \operatorname{tr}\left\{P_{\boldsymbol{\omega}_{\Gamma_{j}}}([0, E]) u_{k}\right\}+\mathrm{e}^{-\frac{t}{2} E} \operatorname{tr}\left\{\mathrm{e}^{-H_{0}} u_{k}\right\} \\
& \leq \operatorname{tr}\left\{P_{\boldsymbol{\omega}_{\Gamma_{j}}}([0, E]) u_{k}\right\}+C_{d, V_{\mathrm{per}}, \delta_{+}} \mathrm{e}^{-\frac{t}{2} E} .
\end{aligned}
$$

Since $\Gamma_{j}$ is a spine of order $2 K$ and $L \in 2 q K \mathbb{N}$, the random operator $H_{\boldsymbol{\omega}_{\Gamma_{j}}}^{(\Lambda)}$ is covariant in the torus $\Lambda$, and we have

$$
\begin{aligned}
\mathbb{E}\left\{\operatorname{tr}\left\{P_{\boldsymbol{\omega}_{\Gamma_{j}}}([0, E]) u_{k}\right\}\right\} & =\frac{1}{\#\left(\Gamma_{j} \cap \Lambda\right)} \sum_{r \in k+\Gamma_{j} \cap \Lambda} \mathbb{E}_{\boldsymbol{\omega}_{\Gamma_{j}}}\left\{\operatorname{tr}\left\{P_{\boldsymbol{\omega}_{\Gamma_{j}}}([0, E]) u_{r}\right\}\right\} \\
& \leq \frac{(2 K)^{d}}{|\Lambda|} U_{+} \mathbb{E}_{\boldsymbol{\omega}_{\Gamma_{j}}}\left\{\operatorname{tr} P_{\boldsymbol{\omega}_{\Gamma_{j}}}([0, E])\right\}
\end{aligned}
$$

for all $E>0$. Combining (3.29), (3.31), and (3.32) we get

$$
\mathbb{E}\left\{\operatorname{tr}\left\{\widetilde{P}_{j} u_{k}\right\}\right\} \leq C_{d, V_{\mathrm{per}}, \delta_{+}, K} \mathrm{e}^{t E^{*}}\left(|\Lambda|^{-1} \mathbb{E}_{\boldsymbol{\omega}_{\Gamma_{j}}}\left\{\operatorname{tr} P_{\boldsymbol{\omega}_{\Gamma_{j}}}([0, E])\right\}+\mathrm{e}^{-\frac{t}{2} E}\right) .
$$

To prove (ii), we take $\left.\left.E_{0} \in\right] 0, \frac{1}{8}\right]$, fix $E^{*}=2 E_{0}$, and require $g \in C^{\infty}(\mathbb{R})$ with $\left|g^{(j)}(E)\right| \leq C E_{0}^{-j}$ for all $E \in \mathbb{R}$ and $j=1,2, \ldots, 2 d+4$, where $C$ is a constant independent of $E$. Appplying [GK2, Theorem 2] as in (3.23), we get

$$
\left\|T_{j, k}\right\| \leq C_{d,\left\|V_{\text {per }}^{-}\right\|, \delta_{+}} u_{-}^{-\frac{1}{2}} E_{0}^{-2 d-3}\left(1+d_{\Lambda}(j, k)\right)^{-2 d-2},
$$

and conclude, similarly to (3.26)

$$
\left(\max _{j \in \widetilde{\Lambda}} \sum_{k \in \widetilde{\Lambda}}\left|\left\|\left|T_{j, k}\right|\right\|_{\infty}^{\frac{1}{2}}\right)^{2} \leq C_{d,\left\|V_{\text {per }}^{-}\right\|, \delta_{+}} u_{-}^{-\frac{1}{2}} E_{0}^{-2 d-3} .\right.
$$


Thus, it follows from (3.21) and (3.35) that

$$
\max _{r \in \widetilde{\Lambda}} \mathbb{E}\left\{\operatorname{tr} P u_{r}\right\} \leq C_{d,\left\|V_{\text {per }}^{-}\right\|, \delta_{+}} u_{-}^{-\frac{3}{2}} E_{0}^{-2 d-3} Q_{\Lambda}(|I|)\left(\max _{j, k \in \widetilde{\Lambda}} \mathbb{E}\left\{\operatorname{tr} \widetilde{P}_{j} u_{k}\right\}\right) .
$$

Note that $H_{\boldsymbol{\omega}_{\Gamma_{j}}}$ would be an Anderson Hamiltonian but for the fact that the random potential is located on $\Gamma_{j}$ instead of $\mathbb{Z}^{d}$. All the results for Anderson Hamiltonians apply to $H_{\boldsymbol{\omega}_{\Gamma_{j}}}$, with the obvious modifications. $H_{\boldsymbol{\omega}_{\Gamma_{j}}}$ is a $2 q K \mathbb{Z}^{d}$ ergodic family of random self-adjoint operators. It has an integrated density of states $N_{\Gamma_{j}}(E)$, defined similarly to (2.33), a continuous function in view of the Wegner estimate (2.24). It follows from (2.33) that for all $E \in \mathbb{R}$ there exists $L(E)$ such that for all boxes $\Lambda=\Lambda_{L}$ with $L \geq L(E)$ we have

$$
|\Lambda|^{-1} \mathbb{E}\left(\operatorname{tr} \chi_{]-\infty, E]}\left(H_{\omega_{\Gamma_{j}}}^{(\Lambda)}\right)\right) \leq 2 N_{\Gamma_{j}}(E) .
$$

$N_{\Gamma_{j}}(E)$ satisfies the Lifshitz tails estimate (2.34), so it follows that given $\left.\left.\eta \in\right] 0, \frac{1}{2}\right]$ there exists $E^{*}(\eta)>0$ such that

$$
N_{\Gamma_{j}} \leq \mathrm{e}^{-E^{-\frac{d}{2}+\eta}} \quad \text { for all } \quad E \in\left[0, E^{*}(\eta)\right] .
$$

We conclude that

$$
\left|\Lambda_{L}\right|^{-1} \mathbb{E}\left(\operatorname{tr} \chi_{]-\infty, E]}\left(H_{\boldsymbol{\omega}_{\Gamma_{j}}}^{\left(\Lambda_{L}\right)}\right)\right) \leq 2 \mathrm{e}^{-E^{-\frac{d}{2}+\eta}} \text { for } E \in\left[0, E^{*}(\eta)\right], L \geq L(E) .
$$

In particular, requiring $8 E_{0} \leq E^{*}(\eta)$ and $L \geq L\left(8 E_{0}\right)$, it follows from (3.33) with $E^{*}=2 E_{0}$ and $E=8 E_{0}$, (3.37), and (3.38), that

$$
\mathbb{E}\left\{\operatorname{tr}\left\{\widetilde{P}_{j} u_{k}\right\}\right\} \leq C_{d, V_{\mathrm{per}}, \delta_{+}, K} \mathrm{e}^{2 t E_{0}}\left(\mathrm{e}^{-\left(8 E_{0}\right)^{-\frac{d}{2}+\eta}}+\mathrm{e}^{-4 t E_{0}}\right) .
$$

We now choose $t$ by $\left(t \geq 2\right.$ since $\left.E_{0} \leq \frac{1}{8}\right)$

$$
\mathrm{e}^{-\left(8 E_{0}\right)^{-\frac{d}{2}+\eta}}=\mathrm{e}^{-4 t E_{0}}, \quad \text { i.e., } \quad t=\frac{1}{4}\left(8 E_{0}\right)^{-1-\frac{d}{2}+\eta},
$$

getting

$$
\begin{aligned}
\mathbb{E}\left\{\operatorname{tr}\left\{\widetilde{P}_{j} u_{k}\right\}\right\} & \leq 2 C_{d, V_{\mathrm{per}}, \delta_{+}, K} \mathrm{e}^{2 t E_{0}} \mathrm{e}^{-4 t E_{0}}=2 C_{d, V_{\mathrm{per}}, \delta_{+}, K} \mathrm{e}^{-2 t E_{0}} \\
& =2 C_{d, V_{\mathrm{per}}, \delta_{+}, K} \mathrm{e}^{-\frac{1}{2}\left(8 E_{0}\right)^{-\frac{d}{2}+\eta}} .
\end{aligned}
$$

Thus, if $8 E_{0} \leq E^{*}(\eta)$ and $L \geq L\left(8 E_{0}\right)$ it follows from(3.36) and (3.42) that

$$
\max _{r \in \widetilde{\Lambda}} \mathbb{E}\left\{\operatorname{tr} P u_{r}\right\} \leq C_{d, V_{\mathrm{per}}, \delta_{+}, K} u_{-}^{-\frac{3}{2}} Q_{\Lambda}(|I|) E_{0}^{-2 d-3} \mathrm{e}^{-\frac{1}{2}\left(8 E_{0}\right)^{-\frac{d}{2}+\eta}} .
$$

It follows that there is $E^{\ddagger}(\eta)=E^{\ddagger}\left(\eta, d, V_{\text {per }}, \delta_{+}, u_{-}, K, \mu_{\Gamma}\right)>0$ such that for $E_{0} \leq E^{\ddagger}(\eta)$ and $L \geq L\left(8 E_{0}\right)$ we get

$$
\max _{r \in \widetilde{\Lambda}} \mathbb{E}\left\{\operatorname{tr} P u_{r}\right\} \leq \mathrm{e}^{-E_{0}^{-\frac{d}{2}+\eta}} Q_{\Lambda}(|I|) \quad \text { for } \quad I \subset\left[0, E_{0}\right],
$$

which is (2.28). Thus (ii) is proven.

To prove (iii), we also assume that $\mu_{\Gamma}$ is Hölder continuous, so (2.24) and (2.25) yield a Wegner estimate that allows the performance of the bootstrap multiscale analysis [GK1, Kl1] for the random Schrödinger operator $H_{\boldsymbol{\omega}_{\Gamma_{j}}}$, and hence for $H_{\boldsymbol{\omega}_{j}^{\perp}}$ by treating $V_{\boldsymbol{\omega}_{j}^{\perp} \backslash \boldsymbol{\omega}_{\Gamma_{j}}}$ in (3.28) as a fixed nonnegative uniformly bounded background 
potential as in GK4. The 'a priori' finite volume estimate required for starting the multiscale analysis is given by [GK4, Proposition 4.3]. It follows that there exists $E_{1}>0$ such that we can perform a bootstrap multiscale analysis for $H_{\omega_{j}^{\perp}}$ (using only the random variables $\boldsymbol{\omega}_{\Gamma_{j}}$ ), the constants being uniform in $j \in \mathbb{Z}^{d}$. In particular, taking $0<E_{0} \leq E_{1}, g=\chi_{\left.]-\infty, E_{0}\right]}$ (in particular, $E^{*}=E_{0}$ ), so $\widetilde{P}_{j}=\chi_{\left.]-\infty, E_{0}\right]}\left(H_{\boldsymbol{\omega}_{j}^{\perp}}\right)$, we conclude that (this follows from the multiscale analysis as in GK1, K11, see also [R]; the argument holds in finite volume) for $L$ large (how large depending on $\left.d, V_{\mathrm{per}}, \delta_{ \pm}, u_{-}, \mu_{\Gamma}, K\right)$

$$
\left|\left\|\left|T_{j, k}\right|\right\|_{2} \leq u_{-}^{-\frac{1}{2}}\left\|\chi_{\Lambda_{\delta_{+}}(j)} \widetilde{P}_{j} \chi_{k}\right\|_{2} \leq C_{d, V_{\mathrm{per}}, \delta_{ \pm}, u_{-}} \mathrm{e}^{-\sqrt{d_{\Lambda}(j, k)}} \quad \text { for } \quad j, k \in \widetilde{\Lambda} .\right.
$$

In particular, give $s>0$, we have

$$
\sum_{k \in \widetilde{\Lambda}} \mathrm{e}^{-s \sqrt{d_{\Lambda}(j, k)}} \leq \sum_{k \in \mathbb{Z}^{d}} \mathrm{e}^{-s|k|^{\frac{1}{2}}}=C_{d, s}<\infty \quad \text { for all } j \in \widetilde{\Lambda} .
$$

Since we also have

$$
\left|\left\|\left|T_{j, k}\right|\right\|\right|_{2}=\left|\left\|| | u _ { j } ^ { \frac { 1 } { 2 } } \widetilde { P } _ { j } \hat { \chi } _ { k } \left|\left\|\left.\right|_{2} \leq u_{-}^{-\frac{1}{2}}\left|\left\|\left|u_{j}^{\frac{1}{2}} \widetilde{P}_{j}\right|\right\|\right|_{2}=u_{-}^{-\frac{1}{2}}\left(\mathbb{E}\left\{\operatorname{tr} \widetilde{P}_{j} u_{j}\right\}\right)^{\frac{1}{2}},\right.\right.\right.\right.
$$

it follows from (3.19), (3.45), (3.46), and (3.47), that for any $\eta \in] 0,1[$ we have

$$
\max _{r \in \widetilde{\Lambda}} \mathbb{E}\left\{\operatorname{tr} P u_{r}\right\} \leq C_{d, V_{\mathrm{per}}, \delta_{ \pm}, u_{-}, \eta} Q_{\Lambda}(|I|)\left(\max _{j \in \widetilde{\Lambda}} \mathbb{E}\left\{\operatorname{tr} \widetilde{P}_{j} u_{j}\right\}\right)^{1-\eta} .
$$

We now consider energies $0<E_{2} \leq E_{3}$; we will fix $E_{3}$ later. It follows from (3.33) with $E^{*}=E_{0}, E=E_{2}$, and $t=\frac{1}{E_{0}}$, that

$$
\mathbb{E}\left\{\operatorname{tr}\left\{\widetilde{P}_{j} u_{k}\right\}\right\} \leq C_{d, V_{\mathrm{per}}, \delta_{+}, K}\left(|\Lambda|^{-1} \mathbb{E}_{\boldsymbol{\omega}_{\Gamma_{j}}}\left\{\operatorname{tr} P_{\boldsymbol{\omega}_{\Gamma_{j}}}\left(\left[0, E_{2}\right]\right)\right\}+\mathrm{e}^{-\frac{E_{2}}{2 E_{0}}}\right) .
$$

Using (2.24) and (2.21), we get

$$
\mathbb{E}\left\{\operatorname{tr}\left\{P_{\boldsymbol{\omega}_{\Gamma_{j}}}\left(\left[0, E_{2}\right]\right)\right\}\right\} \leq C_{E_{3}} Q_{\Gamma}^{\left(m_{d}\right)}\left(E_{2}\right) \leq C_{E_{3}} C_{\mu_{\Gamma}, \alpha, m_{d}} E_{2}^{\alpha},
$$

the constant $C_{E_{3}}$ depending only on $d, V_{\text {per }}, \delta_{+}, u_{ \pm}, K$ and on $E_{3}$. Combining (3.49) and (3.50) we get

$$
\mathbb{E}\left\{\operatorname{tr}\left\{\widetilde{P}_{j} u_{j}\right\}\right\} \leq C_{1}\left(C_{\mu_{\Gamma}} E_{2}^{\alpha}+\mathrm{e}^{-\frac{E_{2}}{2 E_{0}}}\right),
$$

with a constant $C_{1}=C_{d, V_{\mathrm{per}}, \delta_{+}, u_{ \pm}, K, E_{1}, E_{3}}$ and $C_{\mu_{\Gamma}}=C_{\mu_{\Gamma}, \alpha, m_{d}}$.

Let $\beta(s)$ be defined on $[0, \infty[$ by $\beta(0)=0$ and

$$
C_{\mu_{\Gamma}}(\beta(s))^{\alpha}=\mathrm{e}^{-\frac{\beta(s)}{2 s}} \text { for } s>0 .
$$

In particular,

$$
C_{\mu_{\Gamma}}(\beta(s))^{\alpha} \mathrm{e}^{\frac{\beta(s)}{2 s}}=1, \quad \text { i.e., } \quad \frac{\beta(s)}{2 \alpha s} \mathrm{e}^{\frac{\beta(s)}{2 \alpha s}}=\left(2 \alpha s C_{\mu_{\Gamma}}^{\frac{1}{\alpha}}\right)^{-1} .
$$

If

$$
\left(2 \alpha s C_{\mu_{\Gamma}}^{\frac{1}{\alpha}}\right)^{-1} \geq 3, \quad \text { i.e., } \quad 6 \alpha s C_{\mu_{\Gamma}}^{\frac{1}{\alpha}} \leq 1
$$

we have

$$
\frac{\beta(s)}{2 \alpha s} \leq \log \left(2 \alpha s C_{\mu_{\Gamma}}^{\frac{1}{\alpha}}\right)^{-1}, \quad \text { i.e., } \quad \beta(s) \leq 2 \alpha s \log \left(2 \alpha s C_{\mu_{\Gamma}}^{\frac{1}{\alpha}}\right)^{-1} .
$$


We now choose $E_{2}=\beta\left(E_{0}\right)$ and $E_{3}=\beta\left(E_{1}\right)$, and require

$$
E_{0} \leq \widetilde{E}_{1}=\min \left\{E_{1},\left(6 \alpha C_{\mu_{\Gamma}}^{\frac{1}{\alpha}}\right)^{-1}\right\}
$$

It follows from (3.51) and (3.55) that

$$
\mathbb{E}\left\{\operatorname{tr}\left\{\widetilde{P}_{j} u_{j}\right\}\right\} \leq 2 C_{1} C_{\mu_{\Gamma}}\left(2 \alpha E_{0} \log \frac{1}{2 \alpha E_{0} C_{\mu_{\Gamma}}^{\frac{1}{\alpha}}}\right)^{\alpha} .
$$

The estimate (2.30) follows immediately from (3.48) and (3.57). This proves (iii).

\section{REFERENCES}

[BoGKS] Bouclet, J.M., Germinet, F., Klein, A., Schenker,J.: Linear response theory for magnetic Schrödinger operators in disordered media. J. Funct. Anal. 226, 301-372 (2005). doi $10.1090 /$ S0002-9939-04-07431-3

[CL] Carmona, R, Lacroix, J.: Spectral theory of random Schrödinger operators. Boston: Birkhaüser, 1990

[CoGK1] Combes, J.M., Germinet, F., Klein, A.: Poisson statistics for eigenvalues of continuum random Schrödinger operators, Analysis and PDE 3, 49-80 (2010). doi $10.2140 /$ apde.2010.3.49

[CoGK2] Combes, J.M., Germinet, F., Klein, A.: Lifshitz tails estimate for the density of states of the Anderson model, RIMS Kkyroku Bessatsu B27, 1-9 (2011)

[CoH] Combes, J.M., Hislop, P.D.: Localization for some continuous, random Hamiltonians in d-dimension. J. Funct. Anal. 124, 149-180 (1994)

[CoHK1] Combes, J.M., Hislop, P.D., Klopp, F.: Hölder continuity of the integrated density of states for some random operators at all energies. IMRN 4, 179-209 (2003)

[CoHK2] Combes, J.M., Hislop, P.D., Klopp, F.: Optimal Wegner estimate and its application to the global continuity of the integrated density of states for random Schrödinger operators. Duke Math. J. 140, 469-498 (2007)

[GK1] Germinet, F., Klein, A.: Bootstrap multiscale analysis and localization in random media. Commun. Math. Phys. 222, 415-448 (2001). doi 10.1007/s002200100518

[GK2] Germinet, F., Klein, A.: Operator kernel estimates for functions of generalized Schrödinger operators. Proc. Amer. Math. Soc. 131, 911-920 (2003). doi $10.1090 /$ S0002-9939-02-06578-4

[GK3] Germinet, F., Klein, A.: A characterization of the Anderson metal-insulator transport transition. Duke Math. J. 124, 309-351 (2004). doi 10.1215/S0012-7094-04-12423-6

[GK4] Germinet, F., Klein, A.: A comprehensive proof of localization for continuous Anderson models with singular random potentials. J. Eur. Math. Soc. 15, 53-143 (2013). doi $10.4171 / \mathrm{JEMS} / 356$

[GKM] Germinet, F, Klein, A., Mandy, B.: Dynamical delocalization in random Landau Hamiltonians with unbounded random couplings. In Spectral and Scattering Theory for Quantum Magnetic Systems, 87-100, Contemp. Math. 500, Amer. Math. Soc., Providence, RI, 2009. doi $10.1090 /$ conm/500/09822

[HT] Hengartner, W., Theodorescu, R.: Concentration functions. Probability and Mathematical Statistics, No. 20. Academic Press [A Subsidiary of Harcourt Brace Jovanovich, Publishers], New York-London, 1973

[KM1] Kirsch, W., Martinelli, F.: On the ergodic properties of the spectrum of general random operators. J. Reine Angew. Math. 334, 141-156 (1982)

[KM2] Kirsch, W., Martinelli, F. : On the spectrum of Schrödinger operators with a random potential. Commun. Math. Phys. 85, 329-350 (1982)

[K11] Klein, A.: Multiscale analysis and localization of random operators. In Random Schrödinger Operators. Panoramas et Synthèses 25, 121-159, Société Mathématique de France, Paris 2008

[Kl2] Klein, A.: Unique continuation principle for spectral projections of Schrödinger operators and optimal Wegner estimates for non-ergodic random Schrödinger operators. Comm. Math Phys. doi 10.1007/s00220-013-1795-x 
[Klo1] Klopp F.: Internal Lifshits tails for random perturbations of periodic Schrödinger operators. Duke Math. J. 98, 335-396 (1999)

[N] Nakamura, S.: A remark on the Dirichlet-Neumann decoupling and the integrated density of states. J. Funct. Anal. 179, 136-152 (2001)

[PF] Pastur, L., Figotin, A.: Spectra of Random and Almost-Periodic Operators. Heidelberg: Springer-Verlag, 1992

[R] Rojas-Molina, C.: Characterization of the Anderson metal-insulator transport transition for non ergodic operators and application. Ann. Henri Poincaré 13, 1575-1611 (2012). doi 10.1007/s00023-012-0163-2

[W] Wegner, F.: Bounds on the density of states in disordered systems, Z. Phys. B44 9-15 (1981)

(Combes) Centre de Physique Théorique, Aix-Marseille Université et Université du Sud Toulon Var, CNRS UMR 6207, F-83130 La Garde, France

E-mail address: combes@cpt.univ-mrs.fr

(Germinet) Université de Cergy-Pontoise, CNRS UMR 8088, Département de Mathématiques, F-95000 Cergy-Pontoise, France

E-mail address: germinet@math.u-cergy.fr

(Klein) University of California, Irvine, Department of Mathematics, Irvine, CA 92697-3875, USA

E-mail address: aklein@uci.edu 